\title{
Chronic productive cough and extensive cysts in left lung
}

\author{
This article was published in the following Dove Press journal: \\ International Journal of General Medicine \\ II October 201I \\ Number of times this article has been viewed
}

\section{Ali Cheraghvandi \\ Saeid Fallah Tafti \\ Firouzeh Talischi}

Nursing Research and Pulmonary Health Center, Masih Daneshvari Hospital, National Research Institute of Tuberculosis and Lung Diseases, Shahid Beheshti University of Medical Sciences, Tehran, Iran
Correspondence: Saeid Fallah Tafti Masih Daneshvari Hospital, Shahid Bahonar Blvd, DarAbad, Tehran 195584 |452, Iran

Tel +26 I05 050

Emailsaeid_ftafti@yahoo.com
Abstract: Bronchiectasis refers to dilated and thickened airways due to chronic inflammation and infections, with anatomic distortion of the bronchi. Here, we describe a 29-year-old man with a history of multiple hospitalizations for lung infection who presented to the pulmonary clinic with a complaint of worsening chronic productive cough in the previous year. This case presentation prompted a review of the etiologies of bronchiectasis in 291 recent cases admitted to Masih Daneshvari Hospital, Tehran.

Keywords: bronchiectasis, cystic lung disease

\section{Introduction}

Bronchiectasis is a pathologic condition of dilated and thickened airways. It is a consequence of chronic inflammation and infections leading to anatomic distortion and dilation of the bronchi. As a result, many insults have to be considered in its pathogenesis, and the condition is typically considered irreversible. However, studies have shown potential reversibility with early treatment. ${ }^{1}$ The description of bronchiectasis dates back to early 19th century in the writings of Rene Theophile Hyacinthe Laennec. In 1992, with the introduction of bronchography by Jean Athnanse, images of lung destruction were obtained, and nowadays the gold standard for diagnosis is high-resolution computed tomography scan. ${ }^{1}$ The clinical presentation is chronic cough (ie, daily for four or more weeks) with purulent sputum and frequent exacerbations, recurrent chest infections, dyspnea, wheeze, hemoptysis, clubbing, failure to thrive, and growth retardation. Pulmonary function tests show obstruction.

\section{Case report}

The patient was a 29-year-old male from Tehran, with a history of multiple hospitalizations for lung infection who presented to the pulmonary clinic with a complaint of chronic productive cough which had worsened in the previous year. He had been hospitalized two to three times for his respiratory condition and once in childhood when he was 10 or 11 years old. The hospitalizations helped him a little, but his sputum production had gradually increased. Since his last hospitalization, which had been 2 years earlier, he had had persistent sputum production with expectoration two or three times an hour. The patient also complained of shortness of breath and wheezing. He denied fever, chills, hemoptysis, or exposure to tuberculosis. He had had measles in childhood. He denied any other past medical history or surgery. He was up to date 
on his vaccinations, was not on any medications, and denied any drug allergies. He was a nonsmoker and did not use drugs or drink alcohol. He was married and worked in an office. He reported having kept a canary at home for the previous year. He denied any medical illnesses in his family, including lung disease.

On physical examination, he was in no acute distress, with a blood pressure of $110 / 70 \mathrm{mmHg}$, a pulse rate of 72 beats per minute, a respiratory rate of 20 per minute, and an oral temperature of $37^{\circ} \mathrm{C}$. He was alert and orientated. He had no lymphadenopathy or sinus tenderness. Heart sounds S1 and S2 were heard, with no murmurs, rubs, or gallops. On pulmonary examination, breath sounds were hyperresonant on the left side. The abdomen was soft and nontender with no organomegaly. Neurology examination was normal. There was mild clubbing of his nails, but no cyanosis or edema.

Chest x-ray showed extensive pulmonary cysts on the left side, with mediastinal shift to the left. Computed tomography scan of the thorax without contrast showed a normal heart, mediastinum, and pleura, with shift to the left side and hyperaeration of the right lung. There were multiple medium-sized, thick-walled cystic lesions, some containing fluid, in the left lung (mostly in the upper lobe), with volume loss in favor of bronchiectasis. The right lung had normal parenchyma and bronchovascular markings. Laboratory investigations showed a white cell count of $10 \times 10^{3}$ cells $/ \mu \mathrm{L}$ and an erythrocyte sedimentation rate of $17 \mathrm{~mm} /$ hour.

In view of illness since childhood, investigations were undertaken to exclude congenital causes of bronchiectasis. The patient was referred for a sweat chloride test and measurement of immunoglobulin levels. Body plethysmography was ordered with culture of sputum for bacteria, fungi, and mycobacteria. Three consecutive sputum smears for acid-fast bacilli were negative. The etiology of bronchiectasis was most likely measles in childhood. The patient's symptoms responded to ciprofloxacin and an albuterol metered dose inhaler.

Electronic medical records from Masih Daneshvari Hospital, a tertiary referral center, were searched to identify all cases of bronchiectasis and associated conditions from January 2007 until July 2008. In total, 291 cases of bronchiectasis were identified, age range 5-83 years, with 24 cases being younger than 18 years. Fifty cases of old tuberculosis were found, as well as 22 cases of cystic fibrosis, 21 of congenital immune deficiency, and five cases of Kartagener's syndrome. The age range for patients with primary ciliary dyskinesia was 9-25 years and that for immune deficiency was 2-43 years. The charts of the last ten patients admitted with a final diagnosis of bronchiectasis were reviewed. Three were presumed to be due to old tuberculosis. One had a tuberculin skin test of $8 \mathrm{~mm}$. All three were sputum-negative for acid-fast bacilli and polymerase chain reaction. One patient was on antituberculous medication. A 17-year-old patient with Job syndrome was identified. One child aged 5 years presented with foreign body (chicken bone) aspiration.

\section{Discussion}

Infection is the most common etiology of bronchiectasis in developing countries. Vaccinations and use of antibiotics have decreased infection-related pediatric bronchiectasis in developed countries. Bronchiectasis is commonly divided into cystic fibrosis and noncystic fibrosis bronchiectasis. Pulmonary changes can be focal or diffuse, and most commonly involve the lower lobes. Mucosal biopsy reveals infiltration by neutrophils and T lymphocytes. On sputum analysis, increased concentrations of inflammatory elements, including neutrophil elastase, interleukin 8 (a potent chemokine for neutrophils), tumor necrosis factor alpha, and prostanoids have been shown. Increased numbers of neutrophils, neutrophil elastase, myeloperoxidase, tumor necrosis factor alpha, and interleukin 6 have been found in the bronchioalveolar lavage fluid of patients with stable disease compared with controls. ${ }^{1}$ Long term-mortality is associated with advancing age, lower body mass index, dyspnea, lack of vaccination, hypoxemia, hypercapnia, and other functional parameters. $^{4}$

The differential diagnosis for this condition is extensive. Childhood conditions associated with bronchiectasis include bacterial, viral, mycobacterial, and fungal infections, immune deficiency (eg, hypogammaglobulinemia), secondary immune deficiency (eg, acquired immunodeficiency syndrome, immunosuppressant therapy), allergic bronchopulmonary aspergillosis, cystic fibrosis, primary ciliary dyskinesia, pulmonary sequestration, alpha-1 antitrypsin deficiency, Williams-Campbell syndrome, other congenital syndromes, Marfan syndrome, aspiration, gastroesophageal reflux, endobronchial tumor or extrinsic narrowing, toxic inhalation, rheumatic and autoimmune disease, and arsenic exposure. ${ }^{1}$ The main causes of bronchiectasis identified in studies of children have been immune deficiency, congenital abnormalities, aspiration, asthma, toxic inhalation, and idiopathic.

For evaluation of immune deficiency, an antibody response to Hemophilus influenzae Type b capsular polysaccharide vaccine of four times or greater has been suggested. Combined variable immune deficiency is more common in 
adults. Humoral immunity should be evaluated promptly in patients with chronic sinopulmonary infection for early therapeutic considerations. Malabsorption syndrome is also seen in these patients. ${ }^{1-9}$

Cystic fibrosis is a common cause of bronchiectasis, diagnosed by elevated sodium and chloride concentrations on sweat chloride testing in a child with recurrent respiratory infections. Cystic fibrosis affects 1/2500 white and 1/17,000 African-American births in the US. It is an autosomal recessive disease involving the cystic fibrosis transmembrane conductance regulator, with delta-F508 being the most common mutation. ${ }^{1}$ Alpha- 1 antitrypsin deficiency is also seen in older patients with more severe lower zone emphysema. ${ }^{8}$

Computed tomography scoring systems have been devised, and are used every two years as follow-up for the disease. Scores are based on bronchiectasis, airway wall thickening due to inflammation, and mucus plugs. ${ }^{6}$ Cystic fibrosis is diffuse and has a predilection for the upper lobe on computed tomography of the chest. ${ }^{10}$ Consolidation, pulmonary nodules, peribronchial thickening, bronchiectasis/bronchial dilation, and air trapping may be seen. ${ }^{11}$ Multidetector computed tomography allows diagnosis and monitoring of cystic fibrosis at substantially lower radiation doses. ${ }^{12}$

Primary ciliary dyskinesia is also an autosomal recessive disorder with variable penetrance. Mean age at diagnosis is 4.4 years, and the disease affects $1 / 15,000$ to $1 / 40,000$ births. It involves different defects in the ciliary ultrastructure. The most serious form is Kartagener's syndrome, which includes $50 \%$ of cases. It is classically described as showing mirror image arrangement, bronchiectasis, and sinusitis. ${ }^{1-3}$ Foreign body aspiration presents with dyspnea, wheezing, coughing, and atelectasis or hyperinflation on chest $\mathrm{x}$-ray. ${ }^{1}$

\section{Treatment}

Addressing recurrent cough, sinusitis, potential foreign body aspiration, and gastroesophageal reflux are cornerstones of prevention. Antibiotics and bronchodilators have traditionally been used as treatment modalities. The most common organisms involved are $H$. influenzae, Pseudomonas spp, and Streptococcus pneumoniae. The risk of Pseudomonas infection increases as time passes and is a marker of disease severity. ${ }^{4}$

General measures include avoiding smoking or secondhand smoke, adequate nutrition, and immunizations, including yearly influenza vaccines, mobilization of secretions with acetylcysteine, hypertonic saline (shown to be beneficial in cystic fibrosis) or mannitol nebulization (shown to be useful in patients with bronchiectasis, cystic fibrosis, and asthma). Inhaled or systemic corticosteroids can be considered if hyperreactivity exists. Aerosolized recombinant DNAse is used in cystic fibrosis. ${ }^{1-4}$

Chest physiotherapy, including postural drainage, chest wall percussion, and forced expiration techniques, are traditionally utilized. In advanced disease or lack of response to appropriate therapy, surgery is an option to decrease infective episodes and for hemoptysis. Following surgery, patients experience a $42.5 \%-75 \%$ improvement in symptoms. ${ }^{1}$ In one study, macrolides reduced sputum volume, improved lung function, and achieved better control of symptoms, with improved survival. ${ }^{4}$ In cases of inadequate humoral immunity, morbidity and infection rates can be reduced by intravenous or subcutaneous immunoglobulin and aggressive antibiotics and pulmonary hygiene measures. ${ }^{1}$ Live vaccines may be harmful. ${ }^{13}$ Patients with primary ciliary dyskinesia or cystic fibrosis may need specialized treatment.

\section{Acknowledgment}

The authors thank their colleagues at Masih Daneshvari Hospital who helped in preparing this case report.

\section{Disclosure}

The authors report no conflicts of interest in this work.

\section{References}

1. Boren EJ, Teuber SS, Gershwin ME. A review of non-cystic fibrosis pediatric bronchiectasis. Clin Rev Allergy Immunol. 2008;34(2):260-273.

2. Ortega HA, Vega Nde A, Santos BQ, Maia GT. Primary ciliary dyskinesia: considerations regarding six cases of Kartagener syndrome. J Bras Pneumol. 2007;33(5):602-608. Portuguese.

3. Bush A, Chodhari R, Collins N, et al. Primary ciliary dyskinesia: current state of the art. Arch Dis Child. 2007;92(12):1136-1140.

4. Ilowite J, Spiegler P, Chawla S. Bronchiectasis: new findings in the pathogenesis and treatment of this disease. Curr Opin Infect Dis. 2008; 21(2):163-167.

5. Alzeer AH, Masood M, Basha SJ, Shaik SA. Survival of bronchiectatic patients with respiratory failure in ICU. BMC Pulm Med. 2007;10;7:17.

6. Tiddens HA, de Jong PA. Imaging and clinical trials in cystic fibrosis. Proc Am Thorac Soc. 2007;4(4):343-346.

7. Lacy P, Lee JL, Vethanayagam D. Sputum analysis in diagnosis and management of obstructive airway diseases. Ther Clin Risk Manag. 2005;1(3):169-179.

8. Wood AM, Simmonds MJ, Bayley DL, Newby PR, Gough SC, Stockley RA. The TNF alpha gene relates to clinical phenotype in alpha-1-antitrypsin deficiency. Respir Res. 2008;9:52.

9. Detková D, de Gracia J, Lopes-da-Silva S, et al. Common variable immunodeficiency: association between memory B cells and lung diseases. Chest. 2007;131(6):1883-1889.

10. Kennedy MP, Noone PG, Leigh MW, et al. High-resolution CT of patients with primary ciliary dyskinesia. AJR Am J Roentgenol. 2007; 188(5):1232-1238. 
11. Davis SD, Fordham LA, Brody AS, et al. Computed tomography reflects lower airway inflammation and tracks changes in early cystic fibrosis. Am J Respir Crit Care Med. 2007;175(9):943-950.

12. Robinson TE. Computed tomography scanning techniques for the evaluation of cystic fibrosis lung disease. Proc Am Thorac Soc. 2007;4(4):310-315.
13. Skorpinski EW, Kung SJ, Yousef E, McGeady SJ. Diagnosis of common variable immunodeficiency in a patient with primary ciliary dyskinesia. Pediatrics. 2007;119(5):e1203-e1205.

\section{Publish your work in this journal}

The International Journal of General Medicine is an international, peer-reviewed open-access journal that focuses on general and internal medicine, pathogenesis, epidemiology, diagnosis, monitoring and treatment protocols. The journal is characterized by the rapid reporting of reviews, original research and clinical studies across all disease areas.
A key focus is the elucidation of disease processes and management protocols resulting in improved outcomes for the patient.The manuscript management system is completely online and includes a very quick and fair peer-review system. Visit http://www.dovepress.com/ testimonials.php to read real quotes from published authors.

Submit your manuscript here: http://www.dovepress.com/international-journal-of-general-medicine-journal 